\title{
Research on the Heavy Block Grinding Wheel Dynamic Balancing System Based on Fuzzy Control
}

\author{
Ma Haitao ${ }^{1}$, Ma Shufan ${ }^{1}$, Jirigalantu' ${ }^{2}$, Yuying $^{3}$ \\ ${ }^{1}$ Changchun University of Technology, changchun 130012,china \\ ${ }^{2}$ The institute of changchun Optical-mechanical, Changchun,130000,china \\ ${ }^{3}$ Changchun aviation university, changchun, 130000 , china \\ mahaitao@mail.ccut.edu.cn,13685350070@163.com, Jiri7702@sohu.com
}

\begin{abstract}
The balance principle of dynamic balance system and the fuzzy controller design method based on the principle of balance weight are introduced in this paper. In addition, we have discussed the grinding wheel dynamic balance with TMS320F240 as the core system of the hardware circuit and the implementation process of software, Experimental results show that: the effect of the correct balance amount and the grinding wheel unbalance correction offset is good, the vibration drop rate reached $93.2 \%$, has played a good role in inhibition of unbalance, the grinder vibration quantity significantly reduced.

Index Terms - The weight balance, Dynamic balance, the fuzzy control.
\end{abstract}

\section{I . Introduction}

Ideally, the pressure of the grinding wheel rotation and not rotating on the bearing is the same.Due to the uneven material or casting defects,the error of processing and assembly, and the wear of the grinding wheel in the process of operation, making the centrifugal inertia force produced by each tiny particles cannot cancel each other out when the wheel rotates.Centrifugal force through a bearing to the machinery and its basis, it will cause vibration and acting on the bearings of pressure changes, fluctuation is large and making a noise, reduces the grinding quality of workpiece, accelerate the bearing wear, shorten the service life of mechanical,and can cause destructive accidents.Must balance the grinding wheel, therefore, to reach the balance precision grade, or to make the resulting mechanical vibration amplitude fall in the allowed range. The balancing time of the traditional balance machine is long , and it's process is complex. In order to reduce the maintenance workload and improve the grinding accuracy, widely admire on-line dynamic balancing technology now.

\section{II . The Structure and Working Principle of the Balancing Head}

\section{A . The internal structure of the balancing head}

As the actuator,the balancing head is a key part of the balance system.The system selects the method of double weight fixed radius of polar coordinates. The internal structure of the balancing head and the head section as shown in figure 1 and figure2. Using the compact ring structure, the balancing head consist of the driving system (DC motor), the transmission mechanism (the transmission gear), the balance ring gear (weight), etc.The balance gear that generate calibration quality driven by gear,rotation in the two different in-plane
respectively.Dc motor and drive gear are linked together by the precise gear transmission system . Takes advantage of the worm and worm wheel structure, not only improve the load capacity and balance precision of the dc motor, but also provides a self-locking function, and the eccentric gear ring is still able to maintain equilibrium at the end of the balance work. The total transmission ratio for 5664 .

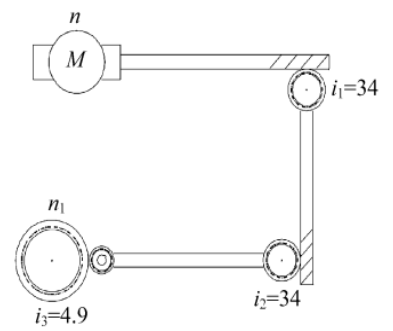

Fig. 1 The internal structure of the balancing head

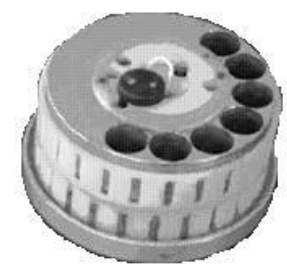

Fig. 2 the cross-section drawn of the balancing head

\section{B. Working Principle of the Balancing Head}

Based on the balance principle of heavy block namely the balancing head method, The balancing head which set up in the end face of the grinding machine spindle synchronous rotate with the grinding wheel.According to the information of the rotor vibration, adjust the position of the two heavy block within the balancing head by the control system to make the correction and the the amount of unbalance offset, achieve the purpose of the grinding wheel balance online.

The driving system of balancing head is composed of two dc permanent magnet motor, which through the worm gear and worm and precision gear train drives the two eccentric gear ring movement, by adjusting the position of the two eccentric gear ring to compensate the balance. The principle of balancing head as shown in figure 3. Set a is centrifugal force produced by the grinding wheel unbalance, $\mathrm{b}$ and $\mathrm{c}$ are 
centrifugal force produced by the two eccentric gear ring, $\mathrm{d}$ is a resultant force of $b$ and $c$. Balance steps: the first step, the control signal drive the two permanent magnet dc motor are foreward or invert at the same time, making the direction of $\mathrm{d}$ and $\mathrm{a}$ in the opposite direction and on the same straight line, namely phase control. The second step, the control signal drive the one permanent magnet $\mathrm{dc}$ motor is forward, and another one is invert, to adjust the included angle of the two eccentric gear ring and the ligature of grinding wheel center point to change the size of the resultant force d, until a and d are equal, namely, amplitude control. The direction of rotation and Angle of the two eccentric gear ring respectively is controlled by fuzzy controller according to the control algorithm.

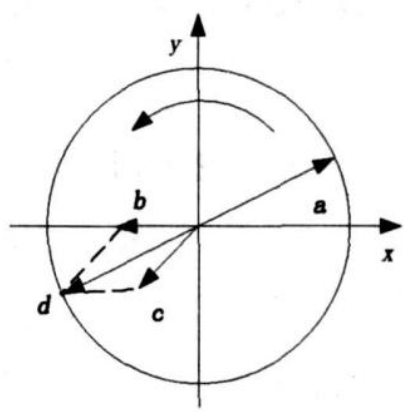

Fig. 3 The principle of balancing head

\section{The Design of Fuzzy Controller}

A. The principle of the fuzzy controller

The basic structure of fuzzy controller is shown in figure 4.

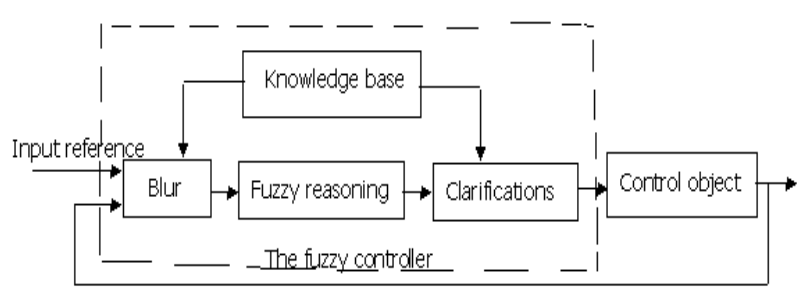

Fig. 4 The structure of fuzzy controller

(1) Blur: Transform the input of accurate quantity into blur amount . Concrete steps as follows: processing the input into the fuzzy controller requires and then the numerical scale transform, make its transformation to the respective fields range and then fuzzy processing, the accurate original input into fuzzy quantity, and use the corresponding fuzzy set to express .

(2) Knowledge base: usually consists of two parts, the database and the fuzzy control rule base, contains the knowledge and the control goal that the specific application field requires.

(3) fuzzy reasoning:the reasoning process is based on the implication relation and rules of the fuzzy logic.

(4) motivation: transform the control amount of fuzzy reasoning getting into the clear amount that actually used in the control.

\section{B. The Design Of Fuzzy Controller}

System uses the acceleration sensor of 8022-01 to measure the vibration of the high-speed rotating wheel,the vibration quantity express by the measured acceleration value indirectly. Set the allowed maximum acceleration for $\mathrm{s}$, actual measured acceleration for $\mathrm{a}$, when a $>\mathrm{s}$, grinding wheel dynamic balance actuator begins to work. By controlling the size and polarity of the armature voltage $u$ and the power-on time $t$ of permanent magnet dc motor for on-line dynamic balance.

(1) Input: detection signal value of the 8022-01 acceleration sensor, with a said that and the language variable as A.

The basic theory of the domain:(c, d)

Quantitative theory of domain: $X=\{0, \ldots 8\}$

Word set: A1, A2, A3, A4, A5

The membership function table of A as shown in table 1 .

TABLE 1 The Membership Function of A

\begin{tabular}{|c|c|c|c|c|c|c|c|c|c|}
\hline & 0 & 1 & $\ddots$ & 3 & 4 & 5 & 6 & 7 & $\Xi$ \\
\hline$A 1$ & 1 & 0.5 & & & & & & & \\
\hline$A 2$ & & 0.5 & 1 & 0.5 & & & & & \\
\hline $\mathrm{A}$ & & & & 0.5 & 1 & 0.5 & & & \\
\hline $\mathrm{A} 4$ & & & & & & 0.5 & 1 & 0.5 & \\
\hline $\mathrm{A} 5$ & & & & & & & & 0.5 & 1 \\
\hline
\end{tabular}
as $\mathrm{U}$.

(2)the output quantity1: voltage $u$, the language variable

The basic theory of the domain:[e, f]

Quantitative theory of domain: $\mathrm{Y}=\{-4,-3,-2,-1,0,+2$, $+3,+4\}$

Word set : $\{$ PB, PS, Z, NS, NB $\}$. Among them, the "P" said dc motor positive spin, "N" said dc motor reverse rotation, "0" said motor stop running, the corresponding fuzzy subset for U1, U2, U3, U4, U5.

The membership function table of $U$ as shown in table 2 .

TABLE 2 The Membership Function of $U$

\begin{tabular}{|c|c|c|c|c|c|c|c|c|c|}
\hline U. & -4 & -3 & -2 & -1 & 0 & 1 & 2 & 3 & 4 \\
\hline ப1 & 1 & 0.5 & & & & & & & \\
\hline ப己 & & 0.5 & 1 & 0.5 & & & & & \\
\hline ப3 & & & & 0.5 & 1 & 0.5 & & & \\
\hline ப4 & & & & & & 0.5 & 1 & 0.5 & \\
\hline ப5 & & & & & & & 0.5 & 1 \\
\hline
\end{tabular}

(3) output 2: electricity time t, the language variable as T. The basic theory of the domain: [g, h] Quantitative theory of domain: $Z=\{0, \ldots 8\}$

Word set: T1, T2, T3, T4, T5

The membership function table of $\mathrm{T}$ as shown in table 3 . 
TABLE 3 The Membership Function of T

\begin{tabular}{|c|c|c|c|c|c|c|c|c|c|}
\hline & 0 & 1 & \ulcorner & 3 & 4 & 5 & 6 & 7 & $B$ \\
\hline$\top 1$ & 1 & 0.5 & & & & & & & \\
\hline$\top 2$ & & 0.5 & 1 & 0.5 & & & & & \\
\hline$\top 3$ & & & & 0.5 & 1 & 0.5 & & & \\
\hline$\top 4$ & & & & & & 0.5 & 1 & 0.5 & \\
\hline$\top 5$ & & & & & & & & 0.5 & 1 \\
\hline
\end{tabular}

(4)The table of control rule : as shown in table 4 and table 5 .

TABLE 4 A $\rightarrow$ U Fuzzy Control Rule

\begin{tabular}{|c|c|c|c|c|l|}
\hline IF & A1 & A2 & A3 & A4 & A5 \\
\hline THEN & U3 & U2/U4 & U2/U4 & U1/U5 & U1/U5 \\
\hline
\end{tabular}

TABLE 5 A $\rightarrow$ T Fuzzy Control Rule

\begin{tabular}{|c|c|c|c|c|c|}
\hline IF & A1 & A2 & A3 & A4 & A5 \\
\hline THEN & T1 & T2 & T3 & T4 & T5 \\
\hline
\end{tabular}

(5) the formation of fuzzy control table

The system is a single input and double output, in the circuit of (A, $\mathrm{U}$ ) have 5 fuzzy control rules and five fuzzy relation can be calculated $R_{I}, R_{2} \ldots R_{5}$.

Get fuzzy relations $R_{1}: R_{1}=A 1 \times U 1$. The general fuzzy relations is $\mathrm{R}=\mathrm{R}_{1} \dot{\mathrm{U}} \mathrm{R}_{2} \ldots \dot{\mathrm{U}} \mathrm{R}_{5}$

Step control algorithm, and any detectable quantity $\mathrm{Ai}=$ $\mathrm{x}$, fuzzy into $\mathrm{x}$ ', by CRI method for fuzzy reasoning, get the fuzzy output to $U^{\prime}=x^{\prime} \circ R$.

Fuzzy judgment: fuzzy judgment by maximum membership degree method for precise control of the amount of U.

In similar way can get the control amount of $\mathrm{T}$ accurately .

\section{The Realization of the Grinding Wheel Dynamic Balance System}

\section{A . the implementation of the hardware}

The grinding wheel dynamic balance system consist by unbalance measurement system (sensor), signal analysis and processing system (controller), the system execution system (balance). First,acceleration sensor of 8022-01 installed on the spindle to test the vibration signal of grinding wheel unbalance caused. After amplification then detection through the low-pass filter and band pass filter and isolate vibration signal of grinding wheel unbalance caused, filter out interference signal, then amplify voltage signal. Make its voltage match with the A/D converter input of the digital signal processor TMS320F240. Change the analog into digital quantity and software filter and digital process, calculate and display the amplitude and phase of unbalance. Finally, sent control commands to the two dc permanent magnet motor according to the control strategy, then through the worm gear and worm and precision gear train drive the two eccentric gear ring movement. Hardware system block diagram as shown in figure 4 , the motor drive circuit diagram as shown in figure 5 .

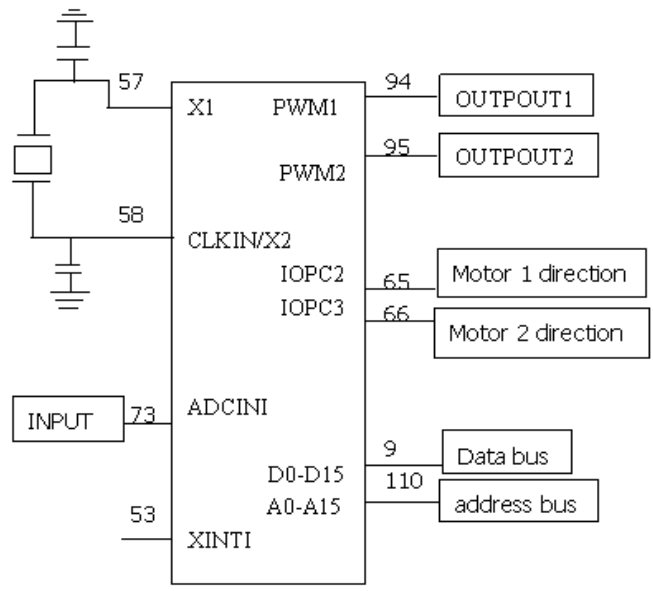

Fig. 4 Hardware system block diagram

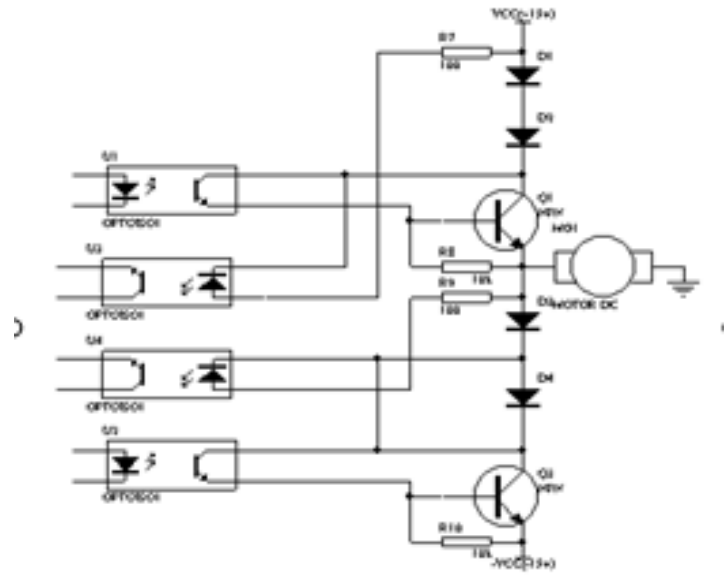

Fig. 5 The motor drive circuit diagram

\section{$B$. Software Implementation}

Write software adopts the DSP instructions of assembly system and modular design, system divided into rotational speed measurement module, system initialization module, vibration measurement module, the system self-checking module, dynamic balance calculation module according to the function. Dynamic balance calculation module is the main component of the system, not only can calculate the amplitude and phase of unbalance, but also can calculate the location of the two balance weight should be moved to under the way of automatic balance. Flow chart is shown in figure 6 . 


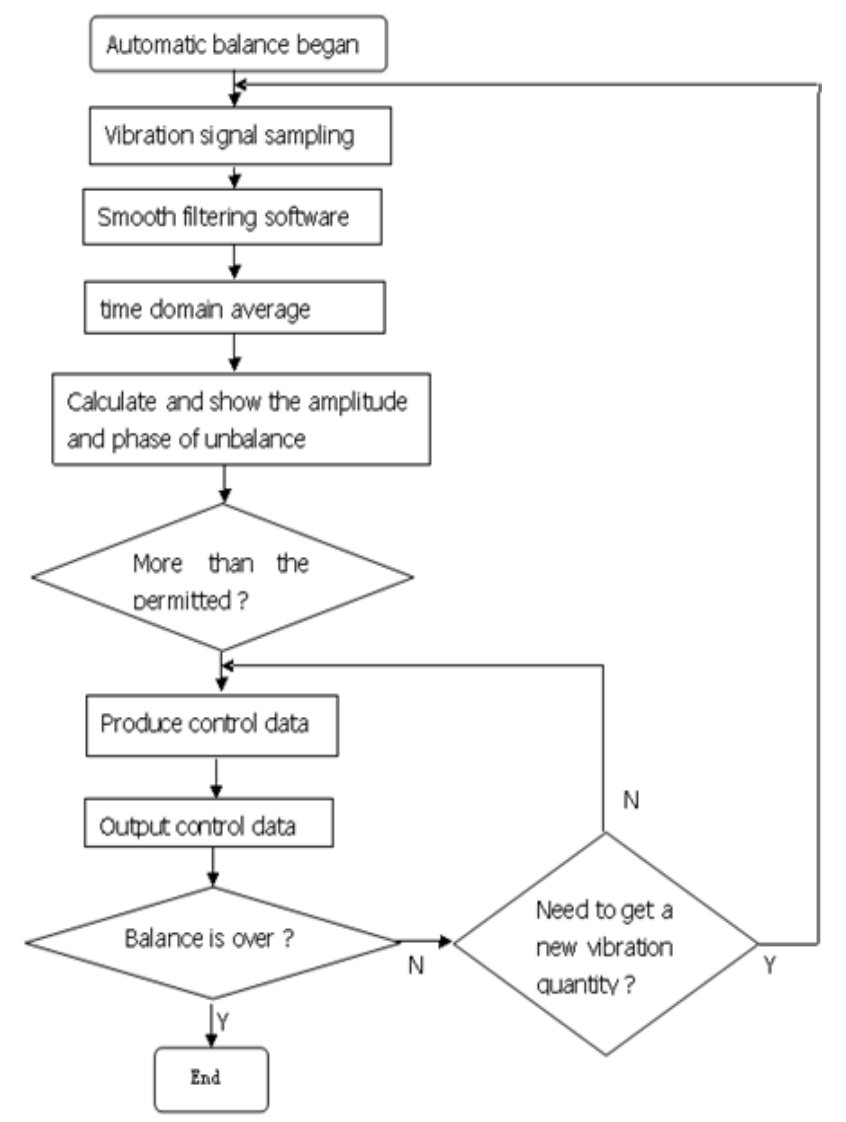

Fig. 6 Flow chart

\section{V . Analysis of Experimental Results}

Test on the outer cylindrical grinder of MG1432, test results as shown in table 6 .
TABLE 6 The Test Results Analysis Table

\begin{tabular}{|l|c|c|c|}
\hline $\begin{array}{c}\text { Control } \\
\text { method }\end{array}$ & $\begin{array}{c}\text { The onginal } \\
\text { vbration } \\
\text { acceleration }\end{array}$ & $\begin{array}{c}\text { after Balance } \\
\text { vibration } \\
\text { acceleration }\end{array}$ & $\begin{array}{c}\text { Vibration } \\
\text { rate drop }\end{array}$ \\
\hline $\begin{array}{l}\text { fuzwy } \\
\text { contol }\end{array}$ & 4.4 & 03 & $932 \%$ \\
\hline
\end{tabular}

From the shows of table 6, this controller has played a good role in inhibition of unbalance, the grinder vibration quantity decreased obviously.

\section{VI . Conclusion}

In this paper, using the principle of weight balance, aim at the difficult problem of establish accurate mathematical model of balancing head, puts forward the fuzzy control algorithm to control the vibration caused by the eccentric wheel. After the test, this system can reduce the amount of unbalance of grinding wheel rotates, reduce the grinder vibration caused by the eccentricity of grinding wheel,improve the quality of the grinding of workpiece .

\section{References}

[1] HaitaoMa, wenYou, WenchaoJia, Based on the weight balance principle of the grinding wheel dynamic balance system modeling method. Journal of changchun university of technology. In March 2006, 27 (7). 21-23.

[2] WenYou,HaitaoMa,WenchaoJia, Modeling and analysis of the grinding wheel dynamic balance measurement and control system. The computer measurement and control. 2006 (8) 14,. 1049-1051.

[3] JianbinZheng,Lingling,WeiZhou,Rotor dynamic balancing steady speed fuzzy control research. Journal of wuhan university of automobile industry. Vol. 21 No. 5.

[4] DongchunWu,ShuangJiang,Parameter self-tuning fuzzy controller design and matlab simulation. Electric welding machine. Vol. 41, No. 5 May 2011.

[5] JiangPing,A PID fuzzy controller. Automation panorama. 2011 (01). 